\title{
Asymptotic Performance Analysis of Two-Way Relaying FSO Networks with Nonzero Boresight Pointing Errors Over Double-Generalized Gamma Fading Channels
}

\author{
Liang Yang, Mohamed-Slim Alouini, and Imran Shafique Ansari
}

\begin{abstract}
In this correspondence, an asymptotic performance analysis for two-way relaying free-space optical (FSO) communication systems with nonzero boresight pointing errors over double-generalized gamma fading channels is presented. Assuming amplify-and-forward (AF) relaying, two nodes having the FSO ability can communicate with each other through the optical links. With this setup, an approximate cumulative distribution function (CDF) expression for the overall signal-to-noise ratio (SNR) is presented. With this statistic distribution, we derive some asymptotic analytical results for the outage probability and average bit error rate. Furthermore, we provide an asymptotic average capacity analysis for high SNR by using the momentsbased method.
\end{abstract}

Index Terms-Double-generalized gamma fading, free-space optical communication, pointing errors, two-way relaying.

\section{INTRODUCTION}

Free space optical (FSO) communication systems have become a hot research topic because of their high bandwidth [1]. Such systems can provide high data rate and are licensefree, which makes them cost-effective and applicable to a lot of applications. However, atmospheric turbulence-induced fading severely degrades FSO link performance.

To overcome this disadvantage, recently the relay-assisted FSO system has received considerable attention. Up to now, a lot of work has investigated the performance of relay-assisted FSO transmission [2]-[6]. However, these works are only available to the one-way relaying systems. In RF wireless communication systems, performance analysis of two-way relaying (TWR) networks has been extensively investigated in the literature since TWR can break through the spectral efficiency loss because of the use of half-duplex in one-way relay channels. In conventional one-way relaying systems, the nodes are generally operated in half-duplex mode, while the TWR scheme can be operating in full-duplex by using the physical-layer network coding (PNC) [7]. In TWR networks, two sources can send their signals simultaneously to the relay in a multiple-access channel, while in the second phase the relay broadcasts it received signal to the two sources. Recently, two-way relay-assisted FSO (TWR-FSO) systems have been considered in [8]-[12]. Using TWR, two FSO nodes can exchange information with each other through the FSO links. More specifically, a TWR-FSO scheme was first proposed in [8], where bit-error rate performance was analyzed. Later, the authors in [9] took pointing errors into consideration and analyzed the system performance of a network-coded coherent TWR-FSO system. More recently, the authors in [9] extended their works to multiple relayed FSO networks [10]-[12]. For instance, single relay selection with amplify-and-forward (AF) considered in [10], partial dual-relay selection with AF studied in [11], and partial dual-relay selection with decode-andforward (DF) investigated in [12].

Over the years, some statistical channel models have been reported to model the irradiance of the atmospheric turbulence for a wide range of turbulence conditions, such as the most widely used log-normal model, $K$, Gamma-Gamma, double Weibull [13], generalized $\mathcal{M}$-distribution [14], and doublegeneralized gamma model (Double GG) [15]. As shown in [15], compared with double Weibull, the proposed Double GG model has a higher accuracy. Under strong turbulence conditions, the results in [15] show that the Double GG has an advantage over the Gamma-Gamma. Later, the performance of FSO networks under Double GG with pointing errors was considered in [16] and [17]. For TWR-FSO systems [8]-[12], the analytical results are only suitable for Gamma-Gamma distribution. On the other hand, in FSO communication systems, pointing errors may appear due to building sway. Boresight and jitter are the two components of these pointing errors. Until now, the performance analysis of the FSO systems with zero boresight pointing errors over various channel models and system setups can be found in a lot of work in the literature, for instance, double GG channels [16][17], log-normal distributed and Gamma-Gamma distributed turbulence [18], average Kdistributed turbulence [19], and $\mathcal{M}$-distributed fading [20], [21]. However, in some cases, the boresight component may be nonzero. Regarding the nonzero boresight pointing error, more details can be found in [22]. In [22], the authors have derived the statistic distribution expressions for the nonzero boresight pointing errors model under the composite log-normal and Gamma-Gamma turbulence-induced fading channels. In [23], the authors presented the ergodic capacity analysis of the FSO systems with nonzero boresight pointing errors over several different atmospheric turbulence channels, for instance, lognormal, Rician-lognormal, and $\mathcal{M}$ distribution. More recently, performance analysis for the FSO networks with nonzero boresight pointing errors under Weibull fading channels was studied in [24].

As per the authors' best knowledge, the performance analysis of the TWR-FSO network with nonzero boresight pointing 
errors over Double GG turbulence channels has not been reported in the literature. Hence, our motivation is to investigate the effect of the nonzero boresight pointing errors on the system performance of a TWR-FSO network under Double GG turbulence channels. In this work, we adopt the coherent detection which has been used in FSO systems to allow the rejection of the background noise and intentional interference and improve the system spectral efficiency [1]. As studied in [25], the coherent FSO systems have a better system performance than the subcarrier intensity modulation (SIM) systems. In this work, an approximate series probability density function (PDF) for the composite Double GG channels is derived. Then, the asymptotic performance analysis is provided to investigate the diversity order and capacity scaling law. Finally, we show some numerical examples to verify our analytical results.

\section{SYSTEM MODEL}

\section{A. TWR-FSO Network}

Consider a TWR-FSO network as shown in Fig. 1 where nodes A and B with FSO ability establish communication via a relay using AF protocol. In this setup, A and B are installed at the top of two different buildings and such a system can be applied in enterprise/campus connectivity. We assume that heterodyne detection and binary phase shift keying (BPSK) modulation are employed. We further assume that perfect synchronization is ensured among the nodes and direct link between A and B is broken. In this network, both A and B are assumed to be equipped with single apertures, while the relay has two apertures for signal reception transmitted from A and B. In this work, it is assumed that our system is corrupted by shot/thermal noise which is modeled as zero-mean additive white Gaussian noise (AWGN) with power spectral density equal to $N_{0}$. To implement the information transmission, two time slots are required. In the first phase, RF signals at $\mathrm{A}$ and $\mathrm{B}$ are converted to optical signals and then are transmitted to the relay. Thus, the resulting signals at the relay can be expressed as [9]

$$
\begin{aligned}
& y_{A R}=\mathcal{R}_{A R} h_{A R} x_{A}+n_{A R}, \\
& y_{B R}=\mathcal{R}_{B R} h_{B R} x_{B}+n_{B R},
\end{aligned}
$$

where $\mathcal{R}_{A R}$ and $\mathcal{R}_{A R}$ are the responsivity of the photodetectors at the relay, $x_{A}$ and $x_{B}$ are the transmitted optical signals with unit energy, $n_{A R}$ and $n_{B R}$ are the AWGN at the relay and $h_{A R}$ and $h_{B R}$ are the channel gains. In (1), the channel gain can be modeled as $h_{A R}=h_{A R}^{a} h_{A R}^{p}$, where $h_{A R}^{a}$ represents the atmospheric turbulence loss and $h_{A R}^{p}$ is the pointing error loss. Note that $h_{A R}^{a}$ and $h_{A R}^{p}$ are independent random variables.

In the second time slot, the relay combines the received optical signal with a local optical signal and then it is focused onto a photodetector. Later, this photodetector helps to convert received optical signals to an electrical signal which is amplified and converted to an optical signal which is retransmitted to $\mathrm{B}$ or A through the FSO links. Thus, the received optical signals at $\mathrm{A}$ and $\mathrm{B}$ are given by

$$
y_{A}=\mathcal{R}_{R A} h_{A R} G_{R A}\left(\mathcal{R}_{B R} h_{B R} x_{B}+n_{B R}\right)+n_{A}
$$

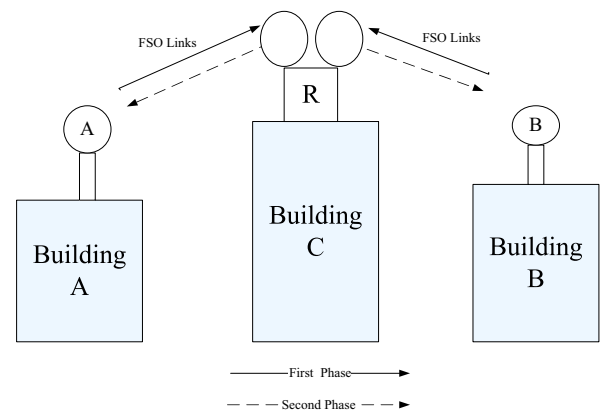

Fig. 1. System diagram of a two-way relaying FSO network

$$
y_{B}=\mathcal{R}_{R B} h_{B R} G_{R B}\left(\mathcal{R}_{A R} h_{A R} x_{A}+n_{A R}\right)+n_{B}
$$

where the amplifying factors are given by $G_{R A}=1 / h_{B R}$ and $G_{R B}=1 / h_{A R}$ for channel state information (CSI)-assisted AF, $n_{A}$ and $n_{B}$ are the AWGN. In (3) and (4), $\mathcal{R}_{R A}$ and $\mathcal{R}_{R B}$ are the photodetector responsivity. Without loss of generality, we assume $\mathcal{R}_{R A}=\mathcal{R}_{R B}=\mathcal{R}_{B R}=\mathcal{R}_{A R}=\mathcal{R}$.

From (3) and (4), the resulting overall signal-to-noise (SNR) of the TWR-FSO system is given by

$$
\gamma_{e 2 e} \approx \frac{\gamma_{1} \gamma_{2}}{\gamma_{1}+\gamma_{2}}
$$

where $\gamma_{1}=\mathcal{R} A_{0} h_{A R} /(q \Delta f)=\frac{\mu}{E\left[h_{A R}\right]} h_{A R}$ [23] and $\gamma_{2}=$ $\mathcal{R} A_{0} h_{B R} /(q \Delta f)=\frac{\mu}{E\left[h_{B R}\right]} h_{B R}$, in which $q, A_{0}$, and $\Delta f$ are the electronic charge, the photodetector area, the noise equivalent bandwidth of an optical wireless communication (OWC) receiver, respectively, $E[\cdot]$ is the expectation operator, and $\mu$ is the average SNR.

\section{B. Channel Statistical Model}

In our work, we use double GG distribution to characterize the turbulence and its PDF of $h_{A R}^{a}$ is given by [15]

$$
\begin{aligned}
f_{h_{A R}^{a}}(h) & =\frac{\beta_{2} p p^{m_{2}-1 / 2} q^{m_{1}-1 / 2}(2 \pi)^{1-(p+q) / 2} h^{-1}}{\Gamma\left(m_{1}\right) \Gamma\left(m_{2}\right)} \\
\cdot & G_{p+q, 0}^{0, p+q}\left(\left.\left(\frac{\Omega_{2}}{h^{\beta_{2}}}\right)^{p} \frac{p^{p} q^{q} \Omega_{1}^{q}}{m_{1}^{q} m_{2}^{p}}\right|_{-} ^{\Delta\left(q: 1-m_{1}\right), \Delta\left(p: 1-m_{2}\right)}\right),
\end{aligned}
$$

where $G_{p, q}^{m, n}(\cdot)$ denotes the Meijers G-function, $\beta_{i}>0, m_{i} \geq$ 0.5 and $\Omega_{i}(i=1,2)$ are the generalized gamma parameters. In (6), $p$ and $q$ are positive integer numbers satisfying $p / q=$ $\beta_{1} / \beta_{2}$ and $\Delta(j: x)=x / j, \ldots,(x+j-1) / j$.

From [22], the PDF of $h_{A R}^{p}$ due to nonzero boresight pointing errors can be expressed as

$$
\begin{aligned}
f_{h_{A R}^{p}}(h) & =\frac{g^{2}}{A_{1}^{g^{2}}} \exp \left(-\frac{s^{2}}{2 \sigma_{s}^{2}}\right) h^{g^{2}-1} \\
& \cdot I_{0}\left(\frac{s}{\sigma_{s}} \sqrt{-2 g^{2} \ln \left(\frac{h}{A_{1}}\right)}\right), 0 \leq h \leq A_{1}
\end{aligned}
$$

where $A_{1}$ is a constant and its value $A_{1}=\operatorname{erf}^{2}(v)$, where $v=$ $\sqrt{\pi / 2} r / z_{b}, r$ is the aperture radius, and $z_{b}$ is the beamwidth. In (7), $g=w_{Z e q} /\left(2 \sigma_{s}\right)$, where $w_{Z e q}$ is the equivalent beam radius at the receiver and $\sigma_{s}$ is jitter standard deviation at the receiver. In (7), $s$ is the boresight displacement and $I_{0}(\cdot)$ 
represents the modified Bessel function of the first kind. Notice that $s=0$ in (7) reduces to the zero boresight pointing error case. By setting $s=0,(7)$ reduces to [18]

$$
f_{h_{A R}^{p}}(h)=\frac{g^{2}}{A_{1}^{g^{2}}} h^{g^{2}-1} .
$$

To the best of our knowledge, there is no analytical result available for the PDF of the combined channel statistical model under the influence of nonzero boresight errors. Recall that $h_{A R}=h_{A R}^{a} h_{A R}^{p}$, the PDF of $h_{A R}$ can be written as

$$
f_{h_{A R}}(h)=\int_{h / A_{1}}^{\infty} \frac{1}{x} f_{h_{A R}^{p}}\left(\frac{h}{x}\right) f_{h_{A R}^{a}}(x) d x .
$$

By substituting (6) and (7) into (9), the PDF of $h_{A R}$ can be obtained as

$$
\begin{aligned}
f_{h_{A R}}(h) & =g^{2} e^{-\frac{s^{2}}{2 \sigma_{s}^{2}}} h^{g^{2}-1} \frac{\beta_{2} p p^{m_{2}-1 / 2} q^{m_{1}-1 / 2}(2 \pi)^{1-(p+q) / 2}}{A_{1}^{g^{2} \Gamma\left(m_{1}\right) \Gamma\left(m_{2}\right)}} \\
& \cdot \int_{h / A_{1}}^{\infty} x^{-\left(g^{2}+1\right)} I_{0}\left(\frac{s}{\sigma_{s}} \sqrt{-2 g^{2} \ln \left(\frac{h}{x A_{1}}\right)}\right) \\
& \cdot G_{p+q, 0}^{0, p+q}\left(\left.\left(\frac{\Omega_{2}}{x^{\beta_{2}}}\right)^{p} \frac{p^{p} q^{q} \Omega_{1}^{q}}{m_{1}^{q} m_{2}^{p}}\right|_{-} ^{\Delta\left(q: 1-m_{1}\right), \Delta\left(p: 1-m_{2}\right)}\right) d x
\end{aligned}
$$

Unfortunately, it is very difficult to evaluate the integral in Eq. (10). Using a transformation of variable, eq. (10) can be rewritten as

$$
\begin{aligned}
& f_{h_{A R}}(h)=e^{-\frac{s^{2}}{2 \sigma_{s}^{2}}} \frac{\beta_{2} p p^{m_{2}-1 / 2} q^{m_{1}-1 / 2}(2 \pi)^{1-(p+q) / 2}}{h \Gamma\left(m_{1}\right) \Gamma\left(m_{2}\right)} \\
& \cdot \int_{0}^{\infty} y e^{-\frac{y^{2}}{2}} I_{0}\left(\frac{s}{\sigma_{s}} y\right) \\
& \cdot G_{p+q, 0}^{0, p+q}\left(\left.W\left(\frac{A_{1}}{h}\right)^{\beta_{2} p} e^{-\frac{\beta_{2} p}{2 g^{2}} y^{2}}\right|_{-} ^{\Delta\left(q: 1-m_{1}\right), \Delta\left(p: 1-m_{2}\right)}\right) d y
\end{aligned}
$$

where $W=\left(\frac{\Omega_{2} p}{m_{2}}\right)^{p}\left(\frac{\Omega_{1} q}{m_{1}}\right)^{q}$. Using $G_{p, q}^{m, n}\left(\left.z^{-1}\right|_{b_{s}} ^{a_{r}}\right)=$ $G_{q, p}^{n, m}\left(\left.z\right|_{1-a_{r}} ^{1-b_{s}}\right)$ [26, eq. (9.31.2)] and applying a series representation of the Meijer G-function when $z \rightarrow 0$ [27, eq.( 07.34.06.0014.01)]

$$
\begin{aligned}
& G_{p, q}^{m, n}\left(\left.z\right|_{b_{1}, \ldots, b_{m}, b_{m+1}, \ldots, b_{q}} ^{a_{1}, \ldots, a_{n}, a_{n+1}, . ., a_{p}}\right)=\sum_{k=1}^{m} \frac{\prod_{j=1, j \neq k}^{m} \Gamma\left(b_{j}-b_{k}\right)}{\prod_{j=n+1}^{p} \Gamma\left(a_{j}-b_{k}\right)} z^{b_{k}} \\
& \cdot \sum_{i=0}^{\infty} \frac{\prod_{j=1}^{n} \Gamma\left(1-a_{j}+b_{k}+i\right) \prod_{j=n+1}^{p}\left(1-a_{j}+b_{k}\right)_{i}\left((-1)^{-m-n+p} z\right)^{i}}{\prod_{j=1}^{m}\left(b_{k}-b_{j}+1\right)_{i} \prod_{j=m+1}^{q} \Gamma\left(1-b_{j}+b_{k}+i\right)}
\end{aligned}
$$

where it requires $p<q, b_{j}-b_{k} \notin \mathbb{Z}$, and $(\cdot)_{n}$ denotes the Pochhamer number defined as $(a)_{n}=a(a+1) \cdots(a+n-1)$ with $(a)_{0}=1$, we have

$$
\begin{aligned}
& f_{h_{A R}}(h)=e^{-\frac{s^{2}}{2 \sigma_{s}^{2}}} \frac{\beta_{2} p p^{m_{2}-1 / 2} q^{m_{1}-1 / 2}(2 \pi)^{1-(p+q) / 2}}{h \Gamma\left(m_{1}\right) \Gamma\left(m_{2}\right)} \sum_{k=1}^{p+q} \\
& \cdot \sum_{i=0}^{\infty} \frac{\prod_{j=1, j \neq k}^{p+q} \Gamma\left(b_{j}-b_{k}\right)(-1)^{i(p-m-n)}}{p+q}\left(\frac{1}{W}\left(\frac{h}{A_{1}}\right)^{\beta_{2} p}\right)^{b_{k}+i} \\
& \prod_{j=1}^{p}\left(b_{k}-b_{j}+1\right)_{i} \\
& \cdot \int_{0}^{\infty} y e^{-\frac{y^{2}}{2}} I_{0}\left(\frac{s}{\sigma_{s}} y\right) e^{\left(b_{k}+i\right) \frac{\beta_{2} p}{2 g^{2}} y^{2}} d y
\end{aligned}
$$

where $b_{j}$ and $b_{k}$ are the values of $1-\Delta\left(q: 1-m_{1}\right), 1-\Delta(p$ : $\left.1-m_{2}\right)$. Using the integral identity [26, eq. (6.643.2)], we can obtain an approximate PDF of the composite channel $h_{A R}$ as

$f_{h_{A R}}(h) \approx e^{-\frac{s^{2}}{2 \sigma_{s}^{2}}} \frac{\beta_{2} p p^{m_{2}-1 / 2} q^{m_{1}-1 / 2}(2 \pi)^{1-(p+q) / 2}}{h \Gamma\left(m_{1}\right) \Gamma\left(m_{2}\right)} \sum_{k=1}^{p+q}$

$$
\begin{aligned}
& \cdot \sum_{i=0}^{J} \frac{\prod_{j=1, j \neq k}^{p+q} \Gamma\left(b_{j}-b_{k}\right)(-1)^{i(p-m-n)}}{\prod_{j=1}^{p+q}\left(b_{k}-b_{j}+1\right)_{i}}\left(\frac{1}{W}\left(\frac{h}{A_{1}}\right)^{\beta_{2} p}\right)^{b_{k}+i} \\
& \cdot \frac{\sigma_{s}}{s} \exp \left(\frac{Q^{2}}{2 \lambda}\right) \lambda^{-0.5} M_{-0.5,0}\left(\frac{Q^{2}}{\lambda}\right)
\end{aligned}
$$

where $J=\left\lfloor\frac{g^{2}}{\beta_{2} p}-b_{k}\right\rfloor$ and $\lfloor x\rfloor$ represents the largest integer not greater than $x$. In (14), $M_{\mu, \nu}(\cdot)$ is the Whittaker function, $\lambda=0.5-\left(b_{k}+i\right) \frac{\beta_{2} p}{2 g^{2}}$, and $Q=s /\left(2 \sigma_{s}\right)$. It should be noted that (14) only holds for $g^{2}>\beta_{2} p b_{k}$ since the integral equation in (13) has such a requirement.

We note that the PDF expression (6) is very involved due to the Meijer G-function. From [28], we know that the asymptotic performance is determined by the behavior of the PDF close to the origin. Therefore, to investigate the asymptotic performance in the next section, we provide the asymptotic PDF expression for $h_{A R}$. From (14), we have the asymptotic PDF expression as

$$
\begin{aligned}
f_{h_{A R}}(h) & \approx e^{-\frac{s^{2}}{2 \sigma_{s}^{2}}} \frac{A}{2 A_{1}^{B}} \prod_{j=1, j \neq k}^{p+q} \Gamma\left(b_{j}^{\prime}-b_{k}^{\prime}\right) Q^{-1} \exp \left(\frac{Q^{2}}{2 U}\right) \\
& \cdot U^{-0.5} M_{-0.5,0}\left(\frac{Q^{2}}{U}\right) h^{B-1}
\end{aligned}
$$

where $U=\left(g^{2}-B\right) /\left(2 g^{2}\right), \quad A=$ $\frac{\beta_{2} p p^{m_{2}-1 / 2} q^{m_{1}-1 / 2}(2 \pi)^{1-(p+q) / 2}}{\Gamma\left(m_{1}\right) \Gamma\left(m_{2}\right) W^{\min \left\{\frac{m_{1}}{q}, \frac{m_{2}}{p}\right\}}}$ and $B=p \beta_{2} \min \left\{\frac{m_{1}}{q}, \frac{m_{2}}{p}\right\}$. In (15), $b_{k}^{\prime}$ and $b_{j}^{\prime}$ are given by

$$
\begin{gathered}
b_{k}^{\prime}=\min \left\{\frac{m_{1}}{q}, \frac{m_{2}}{p}\right\} \\
b_{j}^{\prime} \in\left\{1-\Delta\left(q: 1-m_{1}\right), 1-\Delta\left(p: 1-m_{2}\right)\right\} .
\end{gathered}
$$

In particular, for double-generalized gamma channels with zero boresight pointing errors, the PDF of $h_{A R}$ can be written as [16]

$$
\begin{aligned}
& f_{h_{A R}}(h)=\frac{g^{2} p^{m_{2}-1 / 2} q^{m_{1}-1 / 2}(2 \pi)^{1-(p+q) / 2} h^{-1}}{\Gamma\left(m_{1}\right) \Gamma\left(m_{2}\right)} \\
& \cdot G_{p+q+1,1}^{0, p+q+1}\left(\left.\left(\frac{A_{1}}{h}\right)^{\beta_{2} p} \frac{\Omega_{2}^{p} p^{p} q^{q} \Omega_{1}^{q}}{m_{1}^{q} m_{2}^{p}}\right|_{\kappa_{2}} ^{\kappa_{1}}\right),
\end{aligned}
$$


where $\kappa_{1}=1-\frac{g^{2}}{\beta_{2} p}, \Delta\left(q: 1-m_{1}\right), \Delta\left(p: 1-m_{2}\right)$ and $\kappa_{2}=-\frac{g^{2}}{\beta_{2} p}$. Similarly, from (18), we can obtain an asymptotic PDF expression for double-generalized gamma channels with zero boresight pointing errors as

$$
\begin{aligned}
f_{h_{A R}}(h) \approx & \frac{g^{2} p^{m_{2}-1 / 2} q^{m_{1}-1 / 2}(2 \pi)^{1-(p+q) / 2}}{\Gamma\left(m_{1}\right) \Gamma\left(m_{2}\right)} \\
& \cdot \prod_{j=1, j \neq k}^{p+q+1} \frac{\Gamma\left(b_{j^{\prime}}-b_{k^{\prime}}\right)}{\Gamma\left(1+\frac{g^{2}}{\beta_{2} p}-b_{k^{\prime}}\right)} \frac{1}{\left(A_{1}^{\beta_{2} p} W\right)^{b_{k^{\prime}}}} h^{\beta_{2} p b_{k^{\prime}}-1}
\end{aligned}
$$

where $b_{j^{\prime}}$ and $b_{k^{\prime}}$ are given by

$$
\begin{gathered}
b_{k^{\prime}}=\min \left\{\frac{m_{1}}{q}, \frac{m_{2}}{p}, \frac{g^{2}}{\beta_{2} p}\right\} \\
b_{j^{\prime}} \in\left\{\frac{g^{2}}{\beta_{2} p}, 1-\Delta\left(q: 1-m_{1}\right), 1-\Delta\left(p: 1-m_{2}\right)\right\} .
\end{gathered}
$$

From above analysis, we can see that the exact PDF analysis for the nonzero boresight pointing error case is not possible or challenging. Therefore, asymptotic analysis is presented in this work. In some cases, the asymptotic analysis is more meaningful because some explicit insights can be observed. Therefore, in the following section we adopt the upper bound $\gamma_{e 2 e} \leq \gamma_{u p}=\min \left\{\gamma_{1}, \gamma_{2}\right\}$ to evaluate the system performance. Then, the CDF of $\gamma_{e 2 e}$ can be expressed as

$$
\begin{aligned}
F_{\gamma_{e 2 e}}(\gamma) & =1-\left(1-F_{\gamma_{1}}(\gamma)\right)\left(1-F_{\gamma_{2}}(\gamma)\right) \\
& =F_{\gamma_{1}}(\gamma)+F_{\gamma_{2}}(\gamma)-F_{\gamma_{1}}(\gamma) F_{\gamma_{2}}(\gamma),
\end{aligned}
$$

where $F_{\gamma_{1}}(\gamma)$ and $F_{\gamma_{2}}(\gamma)$ denote the CDFs of $\gamma_{1}$ and $\gamma_{2}$, respectively. With (14), the CDF of $\gamma_{1}$ can be expressed as

$$
\begin{aligned}
& F_{\gamma_{1}}(\gamma) \approx e^{-\frac{s^{2}}{2 \sigma_{s}^{2}}} \frac{\beta_{2} p p^{m_{2}-1 / 2} q^{m_{1}-1 / 2}(2 \pi)^{1-(p+q) / 2}}{\Gamma\left(m_{1}\right) \Gamma\left(m_{2}\right)} \sum_{k=1}^{p+q} \\
& \cdot \prod_{j=1, j \neq k}^{p+q} \sum_{i=0}^{J} \frac{\Gamma\left(b_{j}-b_{k}\right)(-1)^{i(p-m-n)}}{\prod_{j=1}^{p+q}\left(b_{k}-b_{j}+1\right)_{i} \bar{\gamma}_{1}^{\beta_{2} p\left(b_{k}+i\right)}}\left(\frac{1}{W} \frac{1}{A_{1}^{\beta_{2} p}}\right)^{b_{k}+i} \\
& \cdot \frac{\sigma_{s}}{s} \exp \left(\frac{Q^{2}}{2 \lambda}\right) \lambda^{-0.5} M_{-0.5,0}\left(\frac{Q^{2}}{\lambda}\right) \frac{\gamma^{\beta_{2} p\left(b_{k}+i\right)}}{\beta_{2} p\left(b_{k}+i\right)}
\end{aligned}
$$

where $\bar{\gamma}_{1}=\frac{\mu}{E\left[h_{A R}\right]}$.

From (15), the asymptotic cumulative distribution function (CDF) expression for $\gamma_{1}$ can be readily expressed as

$$
\begin{aligned}
& F_{\gamma_{1}}(\gamma) \approx e^{-\frac{s^{2}}{2 \sigma_{s}^{2}}} \frac{A}{2 A_{1}^{B}} \prod_{j=1, j \neq k}^{p+q} \Gamma\left(b_{j}^{\prime}-b_{k}^{\prime}\right) Q^{-1} \exp \left(\frac{Q^{2}}{2 U}\right) \\
& \cdot U^{-0.5} M_{-0.5,0}\left(\frac{Q^{2}}{U}\right) \frac{\gamma^{B}}{B \bar{\gamma}_{1}^{B}} .
\end{aligned}
$$

Similarly, we can obtain CDF expressions $F_{\gamma_{2}}(\gamma)$ for $\gamma_{2}$.

\section{PERFORMANCE ANALYSIS}

Based on the results derived in above section, an asymptotic performance of the system model described in above section is analyzed.

A. Outage Analysis
Generally, outage probability is defined by

$$
P_{\text {out }}=F_{\gamma_{e 2 e}}\left(\gamma_{t h}\right) \text {. }
$$

With (22), (23), and (25), the outage performance of our considered system can be readily evaluated. To investigate the diversity order, we consider the asymptotic outage performance. From (22), the outage probability can be further approximated as

$$
P_{\text {out }} \approx F_{\gamma_{1}}\left(\gamma_{t h}\right)+F_{\gamma_{2}}\left(\gamma_{t h}\right),
$$

where we ignore the negative term in (22) and this is negligible for high SNRs. Therefore, with (24) and (26), we have

$$
P_{\text {out }} \rightarrow \phi\left(\frac{\gamma_{t h}}{\bar{\gamma}_{1}}\right)^{u}+\varphi\left(\frac{\gamma_{t h}}{\bar{\gamma}_{2}}\right)^{w}
$$

where $\phi$ and $\varphi$ are constant terms, and $\bar{\gamma}_{2}=\frac{\mu}{E\left[h_{B R}\right]}$. Let $u=p \beta_{2} \min \left\{\frac{m_{1}}{q}, \frac{m_{2}}{p}\right\}$ and $w=p^{\prime} \beta_{2}^{\prime} \min \left\{\frac{m_{1}^{\prime}}{q^{\prime}}, \frac{m_{2}^{\prime}}{p^{\prime}}\right\}$ where $p^{\prime}$, $\beta_{2}^{\prime}, m_{1}^{\prime}, q^{\prime}, m_{2}^{\prime}$, and $p^{\prime}$ are channel parameters corresponding to the link between the relay and node B. Expression (27) reveals that the diversity gain of our considered system is $\min \{u, w\}$. It should be noted that this diversity order only holds for $g^{2}>\beta_{2} p \min \left\{\frac{m_{1}}{q}, \frac{m_{2}}{p}\right\}$ case, which implies that the Double GG fading has a serious effect on the system performance comparable to the pointing errors at high SNR region. However, for $g^{2}<\beta_{2} p \min \left\{\frac{m_{1}}{q}, \frac{m_{2}}{p}\right\}$ case, the diversity order will be related to both the Double GG fading and pointing error. As mentioned in [22], obtaining an explicit diversity order formula for the nonzero boresight case is very challenging. However, from (19), it is easily observed that the diversity order for the zero boresight case is $\min \left\{\beta_{2} p \min \left\{\frac{m_{1}}{q}, \frac{m_{2}}{p}, \frac{g^{2}}{\beta_{2} p}\right\}, \beta_{2}^{\prime} p^{\prime} \min \left\{\frac{m_{1}^{\prime}}{q^{\prime}}, \frac{m_{2}^{\prime}}{p^{\prime}}, \frac{g^{2}}{\beta_{2}^{\prime} p^{\prime}}\right\}\right\}$.

Notice that the authors in [16] have analyzed the diversity order of Double GG with zero boresight pointing error.

B. BER Analysis

From [28], the bit-error rate (BER) can be evaluated as

$$
P_{e}=E_{X}\left[F_{\gamma_{e 2 e}}\left(\frac{X^{2}}{2}\right)\right],
$$

where $X \sim \mathcal{N}(0,1)$. In this paper, assuming the coherent detection, we can use the conventional digital modulation techniques. Therefore, we can apply (28) most widely used in wireless communication systems to evaluate the error performance of the optical wireless systems.

Substituting (23) into (28), we have

$$
P_{e} \approx I_{1}+I_{2}
$$

where

$$
\begin{aligned}
& I_{1} \approx e^{-\frac{s^{2}}{2 \sigma_{s}^{2}}} \frac{\beta_{2} p p^{m_{2}-1 / 2} q^{m_{1}-1 / 2}(2 \pi)^{1-(p+q) / 2}}{2 \sqrt{\pi} \Gamma\left(m_{1}\right) \Gamma\left(m_{2}\right)} \sum_{k=1}^{p+q} \\
& \cdot \prod_{j=1, j \neq k}^{p+q} \sum_{i=0}^{J} \frac{\Gamma\left(b_{j}-b_{k}\right)(-1)^{i(p-m-n)}}{\prod_{j=1}^{p+q}\left(b_{k}-b_{j}+1\right)_{i} \bar{\gamma}_{1}^{\beta_{2} p\left(b_{k}+i\right)}}\left(\frac{1}{W} \frac{1}{A_{1}^{\beta_{2} p}}\right)^{b_{k}+i} \\
& \cdot \frac{\sigma_{s}}{s} \exp \left(\frac{Q^{2}}{2 \lambda}\right) \lambda^{-0.5} M_{-0.5,0}\left(\frac{Q^{2}}{\lambda}\right) \frac{\Gamma\left(\beta_{2} p\left(b_{k}+i\right)+1\right)}{\beta_{2} p\left(b_{k}+i\right)} .
\end{aligned}
$$


Similarly, using (24), we can express the asymptotical BER as

$$
P_{e} \rightarrow A^{\prime}\left(\frac{1}{\bar{\gamma}_{1}}\right)^{u}+B^{\prime}\left(\frac{1}{\bar{\gamma}_{2}}\right)^{w},
$$

where $A^{\prime}$ and $B^{\prime}$ are constants. As a double check, (31) indicates that the diversity order is still $\min \{u, w\}$.

\section{Asymptotic Average Capacity Analysis}

Generally, regarding the FSO systems, the outage probability or average error rate are the main performance metrics. We note that some research work has considered the average capacity of different FSO setups. For instance, the authors in [29] investigated the ergodic capacity of a FSO network with single apertures at the link ends over two different turbulence channels. Recently, the ergodic capacity of FSO systems for different channel models with nonzero boresight effects was presented in [23].

Since the exact analysis is not available, we resort to an asymptotic capacity analysis and investigate the capacity scaling law. Then, from (5), we can express the average capacity as

$$
\begin{aligned}
E(C) & =E\left[\log _{2}\left(1+\frac{\gamma_{1} \gamma_{2}}{\gamma_{1}+\gamma_{2}}\right)\right]<E\left[\log _{2}\left(1+\min \left\{\gamma_{1}, \gamma_{2}\right\}\right)\right] \\
& <\frac{1}{\ln (2)} E\left[\ln \left(1+\gamma_{1}\right)\right] .
\end{aligned}
$$

As mentioned above, the closed-form PDF of $\gamma_{1}$ is very challenging to be obtained. From [23], at high SNR, the moments-based method can be used to analyze the asymptotic average capacity, namely,

$$
\left.E(C) \approx \frac{\partial}{\partial_{n}} E\left[\gamma_{1}^{n}\right]\right|_{n=0}
$$

where $E\left[\gamma_{1}^{n}\right]$ is the moments.

Since $h_{A R}^{a}$ and $h_{A R}^{p}$ are independent, we have

$$
E\left[\gamma_{1}^{n}\right]=\mu^{n} \frac{E\left[\left(h_{A R}^{a}\right)^{n}\right] E\left[\left(h_{A R}^{p}\right)^{n}\right]}{E^{n}\left[h_{A R}^{a}\right] E^{n}\left[h_{A R}^{p}\right]} .
$$

From [22][23], the moments of $h_{A R}^{p}$ is given by

$$
E\left[\left(h_{A R}^{p}\right)^{n}\right]=\frac{A_{1}^{n} g^{2}}{n+g^{2}} \exp \left(-\frac{n s^{2}}{\left(n+g^{2}\right) 2 \sigma_{s}^{2}}\right) .
$$

Using (6) and [26, Eq.(7.811.4)], we have

$$
E\left[\left(h_{A R}^{a}\right)^{n}\right]=\frac{A_{0}}{\beta_{2} p} \prod_{i=1}^{p+q} \Gamma\left(b_{i}+\frac{n}{\beta_{2} p}\right) W^{\frac{n}{\beta_{2} p}}
$$

where $b_{i}$ has been defined in Eq. (13) and $A_{0}=$ $\frac{\beta_{2} p p^{m_{2}-1 / 2} q^{m_{1}-1 / 2}(2 \pi)^{1-(p+q) / 2}}{\Gamma\left(m_{1}\right) \Gamma\left(m_{2}\right)}$.

Substituting (35) and (36) into (34) yields

$$
E\left[\gamma_{1}^{n}\right]=\frac{\mu^{n} g^{2}\left(1+g^{2}\right)^{n} e^{\frac{n s^{2}}{2 \sigma_{s}^{2}}\left(\frac{1}{1+g^{2}}-\frac{1}{n+g^{2}}\right)} \prod_{i=1}^{p+q} \Gamma\left(b_{i}+\frac{n}{\beta_{2} p}\right)}{g^{2 n}\left(n+g^{2}\right) Y^{n-1} X^{n}}
$$

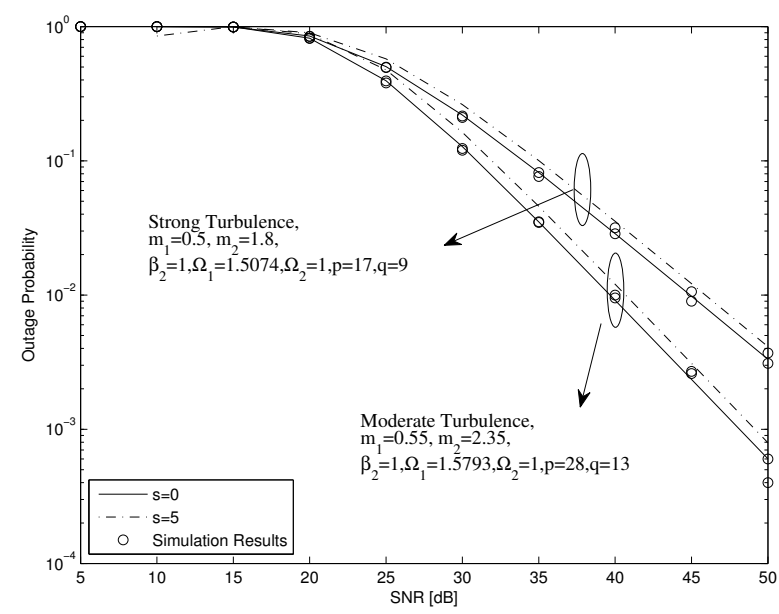

Fig. 2. Outage performance of a relaying FSO network over double generalized gamma channels with non-zero boresight pointing errors.

where $X=\prod_{i=1}^{p+q} \Gamma\left(b_{i}+\frac{1}{\beta_{2} p}\right)$ and $Y=\frac{A_{0}}{\beta_{2} p}$. The first derivative of the moments (37) is given by

$$
\begin{aligned}
& E\left[\gamma_{1}^{n}\right]=\frac{\mu^{n} g^{2}\left(1+g^{2}\right)^{n} e^{\frac{n s^{2}}{2 \sigma_{s}^{2}}\left(\frac{1}{1+g^{2}}-\frac{1}{n+g^{2}}\right)} \prod_{i=1}^{p+q} \Gamma\left(b_{i}+\frac{n}{\beta_{2} p}\right)}{g^{2 n}\left(n+g^{2}\right) Y^{n-1} X^{n}} \\
& \times\left[\ln (\mu)+\ln \left(1+g^{2}\right)+\frac{s^{2}}{2 \sigma_{s}^{2}}\left(\frac{1}{1+g^{2}}-\frac{1}{n+g^{2}}+\frac{n}{\left(n+g^{2}\right)^{2}}\right)\right. \\
& \left.+\frac{1}{\beta_{2} p} \sum_{i=1}^{p+q} \psi\left(b_{i}+\frac{n}{\beta_{2} p}\right)-\ln (X)-\ln (Y)-\ln \left(g^{2}\right)-\frac{1}{n+g^{2}}\right]
\end{aligned}
$$

where $\psi(x)$ is the psi function [26, Eq.(8.3601.1)]. Setting $n=0$ in (38), we can obtain the asymptotic average capacity at high SNR as

$$
\begin{aligned}
E(C) & \approx \frac{Y}{\ln (2)} \prod_{i=1}^{p+q} \Gamma\left(b_{i}\right)\left[\ln (\mu)+\ln \left(1+\frac{1}{g^{2}}\right)-\frac{s^{2}}{2 \sigma_{s}^{2}} \frac{1}{\left(1+g^{2}\right) g^{2}}\right. \\
& \left.+\frac{1}{\beta_{2} p} \sum_{i=1}^{p+q} \psi\left(b_{i}\right)-\ln (X Y)-\frac{1}{g^{2}}\right] .
\end{aligned}
$$

From (39), it is clearly shown that $g$ affects the system capacity significantly. If let $s=0,(39)$ reduces to [16, eq.(35)] corresponding to the zero boresight pointing error case. Moreover, if let $s=0$ and $g \rightarrow \infty$, it corresponds to the zero pointing errors case.

\section{NUMERICAL EXAMPLES}

In this section, we provide some numerical results to show our analysis. Without loss of generality, we assume that $\bar{\gamma}_{1}=$ $\bar{\gamma}_{2}$ and channel parameters for the links are identical. The SNR threshold is set to $\gamma_{t h}=2 \mathrm{~dB}$. Similar to [15][16], we consider two different turbulence conditions, namely, strong and moderate turbulence. For the strong turbulence, we set $m_{1}=0.5, m_{2}=1.8, \beta_{1}=1.8621, \beta_{2}=1, \Omega_{1}=1.5074$, $\Omega_{2}=1, p=17$, and $q=9$. For another, we set $m_{1}=0.55$, $m_{2}=2.35, \beta_{1}=2.1690, \beta_{2}=1, \Omega_{1}=1.5793, \Omega_{2}=1, p=$ 


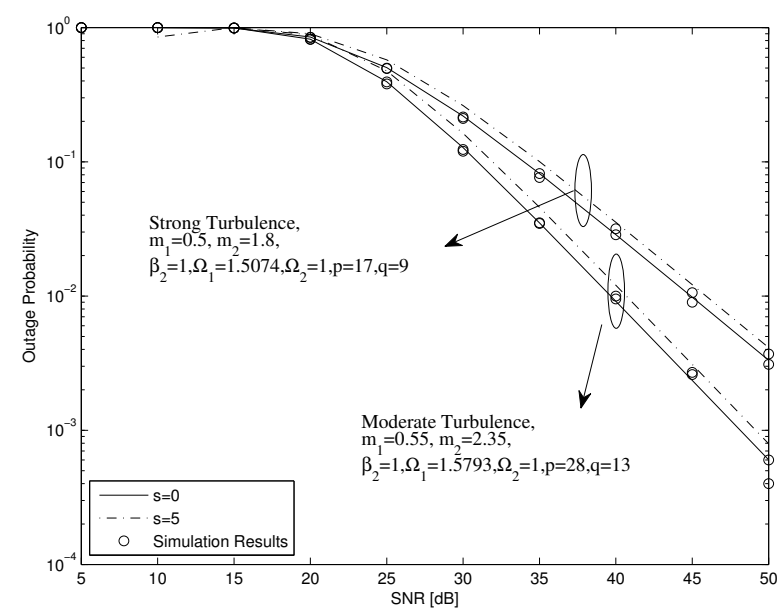

Fig. 3. Outage performance of a relaying FSO network over double generalized gamma channels with non-zero boresight pointing errors.

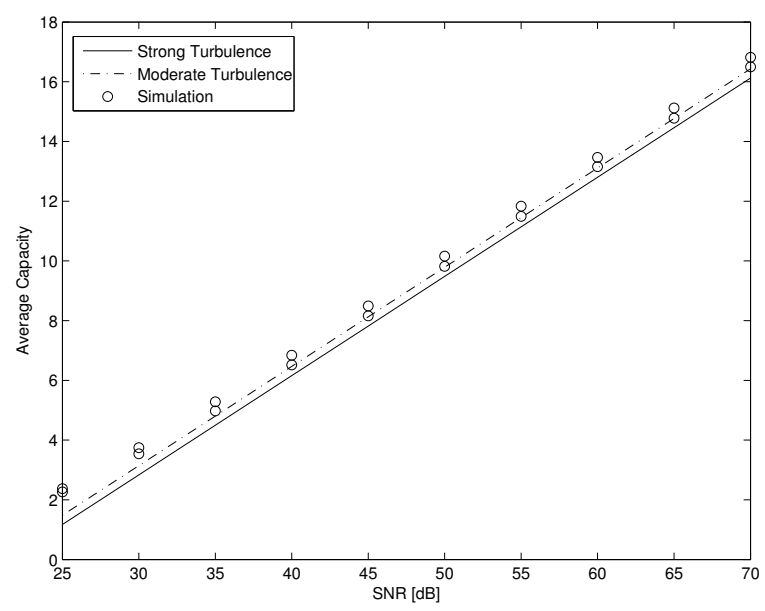

Fig. 4. Capacity of a relaying FSO network over double generalized gamma channels with non-zero boresight pointing errors.

28 , and $q=13$ for the moderate turbulence case. Similarly, we adopt the following system parameters used in some practical terrestrial FSO systems, receiver radius $\mathrm{a}=10 \mathrm{~cm}$, beam radius $\omega_{z}=100 \mathrm{~cm}$, and jitter standard deviation $\sigma_{s}=1.5 \mathrm{~cm}$, see [22] and the references therein. With these system settings, we can obtain $g=5$ and $A_{1}=\left[\operatorname{erf}\left(\sqrt{\pi / 2} a / w_{z}\right)\right]^{2}$.

In Fig. 1, the outage curves for two different values of $s=0,5$ are plotted. From this figure, we can see that both turbulence and non-zero boresight pointing errors affect the system performance. More specifically, for a same $s$, it is clearly observed that the moderate turbulence has a better system performance since the system diversity order depends on $\min \{u, w\}$ for $g^{2}>\beta_{2} p \min \left\{\frac{m_{1}}{q}, \frac{m_{2}}{p}\right\}$, where $u$ and $w$ have been defined in above section. Notice that $g=5$ satisfies this requirement for both turbulence conditions. As expected, it is clearly shown that $s=0$ corresponding to the zero boresight pointing error case has a better system performance. Finally, we can see that the simulation results almost are equal to the asymptotic results at high SNR regions. Using the same parameters used in Fig. 1, the asymptotic BER and capacity are plotted in Fig. 2 and Fig. 3. Similar observations can be obtained.

\section{CONCLUSIONS}

In this correspondence, we investigated the performance of a relaying optical wireless communication system over double generalized gamma atmospheric turbulence channels with non-zero boresight pointing errors. We first derived the approximate statistic distributions of the output SNR. Then, the asymptotic system performance is analyzed and the diversity order is also observed. We further provided asymptotic average capacity analysis to investigate the capacity scaling law.

\section{REFERENCES}

[1] M. Ali. Khalighi and M. Uysal, "Survey on free space optical communication: a communication theroy perspective," IEEE Commmun Surveys Tuts., vol.16, no.4, p.2231-2258, Nov. 2014.

[2] M. Safari and M. Uysal,"Relay-assisted free-space optical communication,'IEEE Trans. Wireless Commun., vol. 7, no. 12, pp. 5441-5449, Dec. 2008.

[3] M. A. Kashani, M. M. Rad, M. Safari, and M. Uysal,"All-optical amplifyand-forward relaying system for atmospheric channels,"IEEE Commun. Lett., vol. 16, no.10, pp. 1684-1686, Oct. 2012.

[4] N. D. Chatzidiamantis, D. S. Michalopoulos, E. E. Kriezis, G. K. Karagiannidis, and R. Schober,"Relay selection protocols for relay-assisted free-space optical systems," IEEE/OSA J. Opt. Commun. Netw, vol. 5, no. 1, pp. 92-103, Jan. 2013.

[5] M. Karimi and M. N.-Kenari,"Free space optical communications via optical amplify-and-forward relaying," IEEE/OSA J. Lightwave Technol., vol. 29, no. 2, pp. 242-248, Jan. 2011.

[6] S. Kazemlou, S. Hranilovic, and S. Kumar,"All-optical multihop freespace optical communication system," IEEE/OSA J. Lightwave Technol., vol. 29, no. 18 , pp. 2663-2669, Sep. 2011.

[7] S. Zhang, S-C Liew, and L. Lu, "Physical Layer Network Coding Schemes over Finite and Infinite Fields, IEEE GLOBECOM 2008, New Orleans, LO, pp. 1-6, Nov. 2008

[8] Y. Tang, X. Zhou, Z. Zhang, and Q. Tian, "Performance analysis of a twoway network-coded free space optical relay scheme over strong turbulence channels," in Proc. Veh. Technol. Conf., San Francisco, CA, USA, Sep. 2011, pp. 1-5.

[9] P. Puri, P. Garg, and M. Aggarwal, "Outage and error rate analysis of network-coded coherent TWR-FSO systems,"IEEE Photon. Technol. Lett., vol.26, no.18, p.1797-1880, Sep. 2014.

[10] P. Puri, N. D. Chatzidiamantis, P. Garg, M. Aggarwal, and G. K. Karagiannidis, "Two-Way relay selection in multiple relayed FSO networks,"IEEE Wireless Commun Lett., vol. 4, no. 5, pp. 485-488, Oct. 2015.

[11] P. Puri, P. Garg, and M. Aggarwal, "Partial dual-relay selection protocols in two-way relayed FSO networks,'IEEE/OSA J. Lightwave Technol., vol. 33, no. 21, pp. 4457-4463, Nov. 2015.

[12] P. Puri, P. Garg, and M. Aggarwal, "Asymptotic analysis of TWR assisted FSO links with partial dual-relay selection,"IEEE Commun Lett., vol. 19, no. 5, pp. 879-882, May 2015.

[13] P. Wang, T. Cao, L. Guo, R. Wang, Y. Yang,"Performance analysis of multihop parallel free-space optical systems over exponentiated Weibull fading channels,"IEEE Photonics Journal., vol. 7, no. 1, Feb 2015.

[14] A. J.-Navas, J. M. G.-Balsells, J. F. Paris, and A. P.-Notario,"A unifying statistical model for atmospheric optical scintillation," in Numerical Simulations of Physical and Engneering Processed, J. Awrejcewicz, E.d., Intech, 2011, ch.8.

[15] M. A. Kashani, M. Uysal, and M. Kavehrad,"A novel statistical channel model for turbulence-induced fading in free-space optical systems,'IEEE/OSA J. Lightwave Technol., vol. 33, no. 11, pp. 2303-2312, June 2015.

[16] H. AlQuwaiee, I. S. Ansari, and M.-S. Alouini, "On the performance of free-space optical communication systems over double generalized Gamma channel,'IEEE J. Sel. Areas Commun., vol. 33, no. 9, pp. 18291840 , Sep 2015. 
[17] H. AlQuwaiee, I. S. Ansari, and M.-S. Alouini, "On the maximum and minimum of double generalized Gamma variates with applications to the performance of free-space optical communication systems,"IEEE Trans. Veh. Technol., vol. 65, no. 11, pp. 8822-8831, Nov. 2016.

[18] A. A. Farid and S. Hranilovic, "Outage capacity optimization for free space optical links with pointing errors, IEEE/OSA J. Lightw. Technol., vol. 25, pp. 1702-1710, Jul. 2007.

[19] H. G. Sandalidis, T. A. Tsiftsis, G. K. Karagiannidis, and M. Uysal, "BER performance of FSO links over strong atmospheric turbulence channels with pointing errors, IEEE Commun. Lett., vol. 12, no. 1, pp. 44-46, Jan. 2008.

[20] H. Samimi and M. Uysal, "End-to-end performance of mixed RF/FSO transmission systems, IEEE/OSA J. Opt. Commun. Netw., vol. 5, no. 11, pp. 1139-1144, Nov. 2013.

[21] A. J. Navas, J. M. G. Balsells, J. F. Paris, M. C. Vzquez, and A. P. Notario, "Impact of pointing errors on the performance of generalized atmospheric optical channels, Opt. Exp., vol. 20, no. 11, pp. 12550-12562, May 2012.

[22] F. Yang, J. Cheng, and T. A. Tsiftsis, "Free-space optical communication with nonzero boresight pointing errors," IEEE Trans. Commun., vol. 62, no. 2, pp. 713-725, Feb. 2014.

[23] I. S. Ansari, M.-S. Alouini, and Julian Cheng,"Ergodic capacity analysis of free-space optical links with nonzero boresight pointing errors,"IEEE Trans. Wireless Commun., vol. 14, no. 8, pp. 4248-4264, Aug. 2015.

[24] P. Wang, T. Cao, L. Guo, X. Liu, H.a Fu, R. Wang, and Y. Yang, "Multihop FSO over exponentiated Weibull fading channels with nonzero boresight pointing errors,'IEEE Photon. Technol. Lett., vol.28, no.16, p.1747-1750, Aug. 2016.

[25] M. Niu, J. Cheng, and J. F. Holzman,"Error rate performance comparison of coherent and subcarrier intensity modulated optical wireless communications," J. Opt. Commun. Netw., vol. 5, no. 6, pp.554-564, June 2013.

[26] I. S. Gradshteyn and I. M. Ryzhik, Table of Integrals, Series, and Products, 6th ed. San Diego, CA:Academic, 2000.

[27] "The Wolfram Functions Site." [Online]. Available: http://functions.wolfram.com.

[28] Y. Zhao, R. Adve, and T. J. Lim, "Symbol error rate of selection amplifyand-forward relay systems," IEEE Commun. Lett., vol. 10, no. 11, pp. 757-759, Nov. 2006.

[29] H. E. Nistazakis, E. A. Karagianni, A. D. Tsigopoulos, M. E. Fafalios, and G. S. Tombras, "Average capacity of optical wireless communication systems over atmospheric turbulence channels,'IEEE/OSA J. Lightwave Technol., vol. 27, no. 8, pp. 974-978, Apr. 2009. 